\title{
Histological validation of a new CMR T1-mapping- based protocol to improve accuracy for fibrosis assessment in patients with aortic stenosis
}

\author{
Vassilis Vassiliou ${ }^{1,2^{*}}$, Katharina Wassilew ${ }^{3}$, George Asimakopoulos $^{6}$, Anthony de Souza ${ }^{6}$, Cesare Quarto ${ }^{6}$, \\ Ee Ling Heng ${ }^{1,2}$, Claire E Raphael ${ }^{1,2}$, Bruce S Spottiswoode ${ }^{4}$, Andreas Greiser ${ }^{4}$, Evangelia Nyktari ${ }^{1}$, \\ Francisco Alpendurada', David Firmin ${ }^{1,2}$, Andrew Jabbour ${ }^{5}$, John Pepper ${ }^{6,2}$, Dudley J Pennell ${ }^{1,2}$, Peter Gatehouse ${ }^{1,2}$, \\ Sanjay Prasad ${ }^{1,2}$
}

From 19th Annual SCMR Scientific Sessions

Los Angeles, CA, USA. 27-30 January 2016

\section{Background}

Short 11-heart beat (11 HB) MOLLI have been proposed as a non-invasive method for the assessment of overall and diffuse fibrosis, but histological correlation has only been modest. We investigated an $11 \mathrm{HB}$ MOLLI protocol with incremental acquisition of basal and mid-level images as a means for obtaining a global value of extracellular volume fraction (ECV). We validated this against intraoperative myocardial biopsies in patients with aortic stenosis.

\section{Methods}

Ten patients ( 8 male, age $73 \pm 7$ years) with aortic stenosis (AS) scheduled for surgical valve replacement (3 with coronary artery disease) underwent CMR at $1.5 \mathrm{~T}$ (Magnetom Avanto, Siemens Healthcare) with native 5 (3)3 and post-gadolinium 4(1)3(1)2 T1 maps (Siemens prototype WIP 448B). Gadolinium $0.1 \mathrm{mmol} / \mathrm{kg}$ was administered, and post-gadolinium T1 maps were taken 15 later. The hematocrit was measured in an approved biochemistry laboratory on the same day. The MOLLI sequence was acquired twice at a single basal short-axis left-ventricular level, and twice at a single mid-ventricular level. Regions of interest were drawn in the septum at both levels to acquire T1 values. ECV calculation utilized an increasing number of maps as shown in table 1 , using $\mathrm{ECV}=(1 \text {-hematocrit })^{*}[(1 / \mathrm{T} 1$ myocardium post contrast-1/T1 myocardium native $)] /[(1 / \mathrm{T} 1$ blood post contrast-1/T1 blood native)].

${ }^{1}$ CMR, Royal Brompton Hospital, London, United Kingdom Full list of author information is available at the end of the article
The following aspects of the methods are proposed as novel steps:

1) "Trucut" biopsy taken intraoperatively from the apical anterior/ lateral wall through the epicardium to allow histological characterization of the full myocardial wall.

2) A CMR imaging model including incrementally more acquisitions was applied as shown in Figure 1.

The myocardial biopsies were fixed in warm buffered formalin. Analysis of formalin-fixed paraffin-embedded, transmural myocardial biopsies of the left ventricle was performed on hematoxylin/eosin and Picrosirius Redstained 3-micron-thick sections by a blinded experienced cardiac pathologist. Images were analysed using a purpose-built software to determine the extent of overall and reactive interstitial fibrosis.

\section{Results}

When only one mid-level MOLLI was taken correlation with histology was modest $\left(R^{2}=0.36\right.$; Figure 2, panel $A$, model A). Increasing the number of image acquisitions and including the basal level increased the correlation significantly: $R^{2}=0.76$ when the average of a single basal and single mid-level image was used for ECV (C, model E) and importantly $\mathrm{R}^{2}=0.83$ when acquisition was repeated at both basal and mid-levels (panel D, model F).

\section{Conclusions}

We have confirmed that ECV can provide accurate correlation with histological overall fibrosis. This work has 


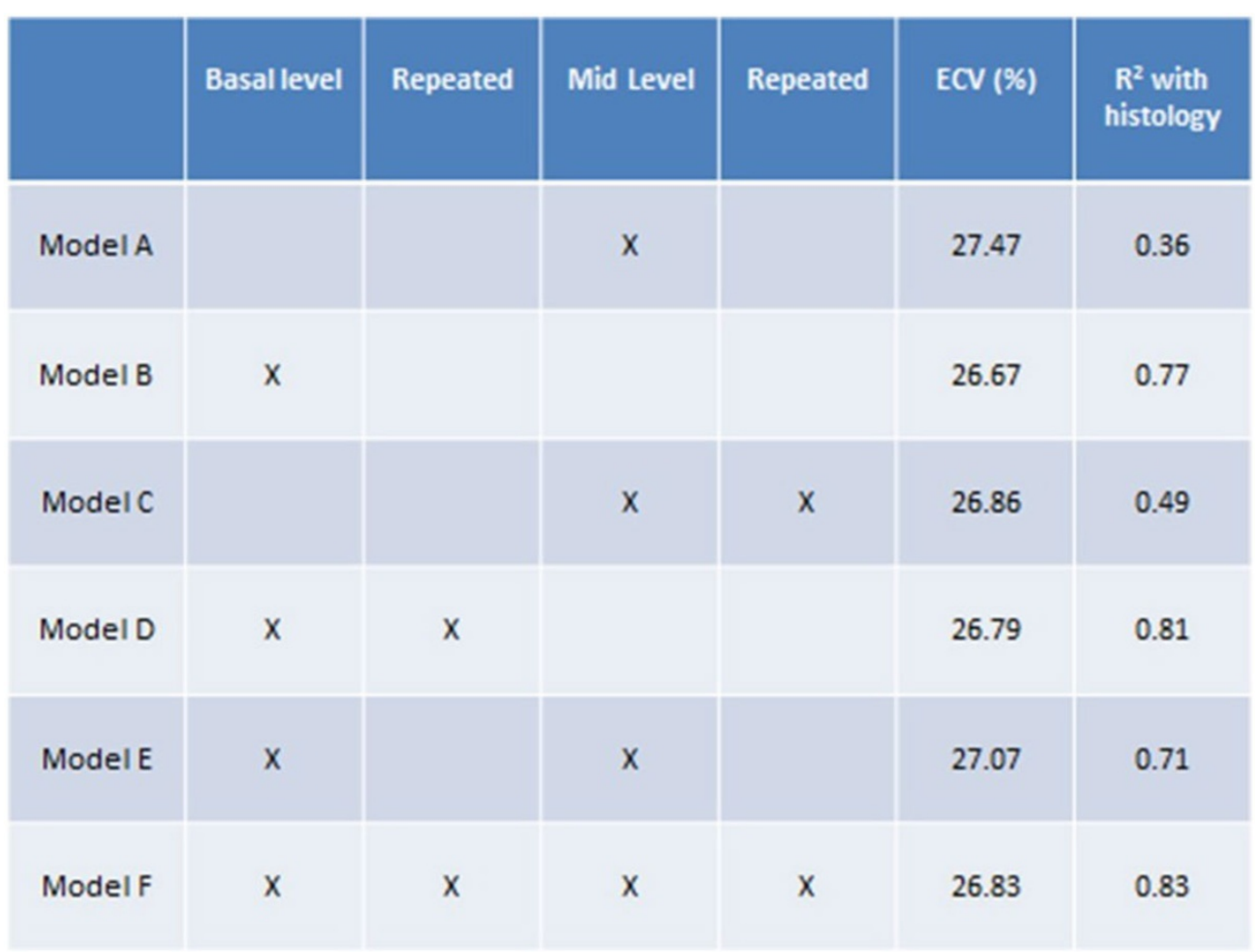

Figure 1 Calculated ECV values using incremental MOLLI image acquisitions for each patient allowing convergence to the true global ECV.

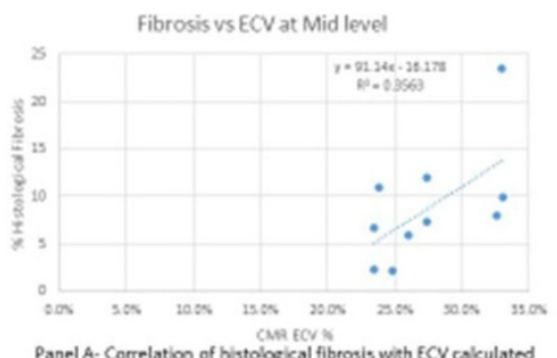

Panel A. Correlation of histological fibrogis with ECV cakulated from CMR at one mid level image

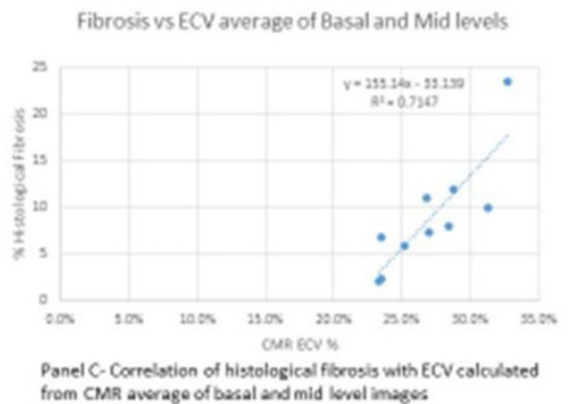

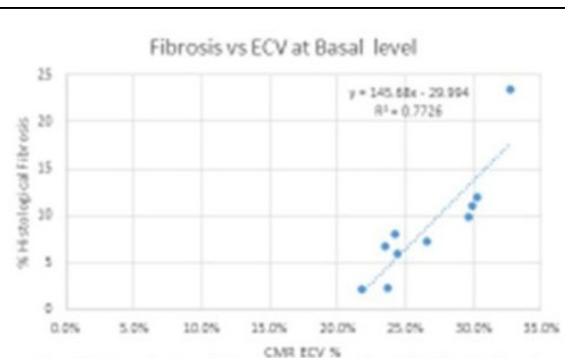

Panel B. Correlation of histological fibrosis with ECV calculated from CMR at one basal level image

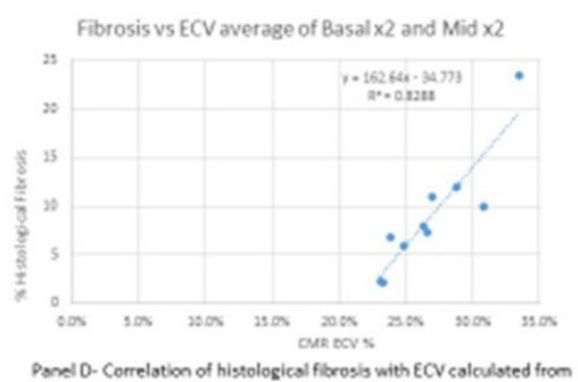

Panel D- Correlation of histolosical florosis with ECV calculated from CMR awerage of two basal \& two mid level images.

Figure 2 Correlation between CMR-calculated ECV and histologically identified fibrosis. The correlation proportionally increased as more levels and repeat sequences were included. Panel A top left: correlation with one mid-level, model A. Panel B top right: correlation with one basal level, model B. Panel C bottom left: correlation with average ECV obtained from the average of one basal and one mid-image, model E. Panel D bottom right: correlation with average ECV obtained from a total of two basal and two mid-level ECV, model F. 
demonstrated that the accuracy increases when MOLLI sequences are acquired at both basal and mid-ventricular levels, and further improves if repeated at each level. We conclude that an average T1 over 2 basal and 2 mid-ventricular level acquisitions improves correlation with histology in aortic stenosis patients when using 11 HB MOLLI.

\section{Authors' details}

${ }^{1}$ CMR, Royal Brompton Hospital, London, United Kingdom. ${ }^{2}$ National Heart and Lung Institute, Imperial College London, London, United Kingdom.

${ }^{3}$ Department of Cardiothoracic and Vascular Surgery, Deutsches Herzzentrum Berlin, Cardiac Pathology Unit, Berlin, Germany. ${ }^{4}$ Siemens Healthcare,

Erlangen, Germany. ${ }^{5}$ St Vincent's Hospital, Sydney, NSW, Australia.

${ }^{6}$ Cardiothoracic Surgery, Royal Brompton Hospital, London, United Kingdom.

Published: 27 January 2016

doi:10.1186/1532-429X-18-S1-Q56

Cite this article as: Vassiliou et al:. Histological validation of a new CMR

T1-mapping-based protocol to improve accuracy for fibrosis assessment in patients with aortic stenosis. Journal of Cardiovascular Magnetic

Resonance 2016 18(Suppl 1):Q56.

Submit your next manuscript to BioMed Central and take full advantage of:

- Convenient online submission

- Thorough peer review

- No space constraints or color figure charges

- Immediate publication on acceptance

- Inclusion in PubMed, CAS, Scopus and Google Scholar

- Research which is freely available for redistribution

Submit your manuscript at www.biomedcentral.com/submit
C Biomed Central 